\title{
Exploiting Contextual Information for Image Re-ranking
}

\author{
Daniel Carlos Guimarães Pedronette and Ricardo da S. Torres \\ RECOD Lab - Institute of Computing - University of Campinas \\ CEP 13083-970, Campinas/SP - Brazil
}

\begin{abstract}
This paper presents a novel re-ranking approach based on contextual information used to improve the effectiveness of ContentBased Image Retrieval (CBIR) tasks. In our approach, image processing techniques are applied to ranked lists defined by CBIR descriptors. Conducted experiments involving shape, color, and texture descriptors demonstrate the effectiveness of our method.
\end{abstract}

\section{Introduction}

Technological improvements in image acquisition and the decreasing cost of storage devices have enabled the dissemination of large image collections. In this scenario, there is the need of methods for indexing and retrieving these data. One of the most common approaches to support image searches relies on the use of Content-Based Image Retrieval (CBIR) systems.

Basically, given a query image, a CBIR system aims at retrieving the most similar images in a collection by taking into account image visual properties (such as, shape, color, and texture). Collection images are ranked in decreasing order of similarity, according to a given image descriptor. However, in general, these approaches perform only pairwise image analysis and compute similarity (or distance) measures considering only pair of images, ignoring the rich information encoded in the relations among several images.

Some post-processing methods have been proposed for improving effectiveness of information retrieval tasks [5, 18,17,9]. Efforts were put on post-processing the similarity scores by analyzing the relations among all documents in a given collection. The influence among shape similarities is analized in [18, using Markov chains to perform a diffusion process on a graph formed by a set of shapes, where the influences of other shapes are propagated. An unsupervised clustering algorithm is used in [5], aiming to capture the manifold structure of the image relations by defining a neighborhood for each data point in terms of a mutual k-nearest neighbor graph. A graph transduction learning approach is introduced in 17. The algorithm computes the shape similarity of a pair of shapes in the context of other shapes. In [9], a distance optimization algorithm has been proposed. The objective is to cluster shapes by taking into account the similariy among ranked lists. Distances between shapes are updated based on created clusters aiming at improving the retrieval effectiveness. 
Recently, contextual information have also been considered for improving the effectiveness of image retrieval [4,10,12]. The objective of these methods is somehow mimic the human behavior on judging the similarity among objects by considering specific contexts. More specifically, the notion of context can refer to updating image similarity measures by taking into account information encoded on the ranked lists defined by a CBIR system [12].

In this paper we present a new post-processing method that re-ranks images by taking into account contextual information. We propose a novel approach for retrieving contextual information, by creating a gray scale image representation of distance matrices computed by CBIR descriptors. The gray scale image is constructed for k-nearest neighbor of a query and analyzed using image processing techniques. The use of image processing techniques for contextual information representation and processing is the main novelty of our work. We believe that our strategy opens a new are of investigation related to the used of image processing approaches for image re-ranking in CBIR systems.

We evaluated the proposed method on shape, color, and texture descriptors. Experimental results demonstrate that the proposed method can be used in several CBIR tasks and yields better results in terms of effectiveness performance than various post-processing algorithms recently proposed in the literature.

\section{A Re-ranking Method Based on Contextual Information}

\subsection{Contextual Information Representation}

Let $\mathcal{C}=\left\{i m g_{1}, i m g_{2}, \ldots, i m g_{N}\right\}$ be an image collection and let $\mathcal{D}$ be an image descriptor that deinfes a distance function $\rho: \mathcal{C} \times \mathcal{C} \rightarrow \mathbb{R}$, where $\mathbb{R}$ denotes real numbers. Consider $\rho(X, Y) \geq 0$ for all $(X, Y)$ and $\rho(X, Y)=0$ if $X=Y$. The distance $\rho\left(i m g_{i}, i m g_{j}\right)$ among all images $i m g_{i}, i m g_{j} \in \mathcal{C}$ can be computed to obtain an $N \times N$ distance matrix $A$.

Our goal is to represent the distance matrix $A$ as a gray scale image (named context image $\hat{I}$ ) and analyse this image for extracting contextual information. For this representation we consider two reference images $i m g_{i}, i m g_{j} \in \mathcal{C}$.

Let the context image $\hat{I}$ be a gray scale image defined by the pair $\left(D_{I}, f\right)$, where $D_{I}$ is a finite set of pixels (points in $\mathbb{N}^{2}$, defined by a pair $(x, y)$ ) and $f: D_{I} \rightarrow \mathbb{R}$ is a function that assigns to each pixel $p \in D_{I}$ a real number. We define the values of $f$ function in terms of the distance function $\rho$ (encoded into matrix $A$ ) and reference images $i m g_{i}, i m g_{j} \in \mathcal{C}$.

Let $R_{i}=\left\{i m g_{i_{1}}, i m g_{i_{2}}, \ldots, i m g_{i_{N}}\right\}$ be the ranked list defined by matrix $A$ considering the reference image $i m g_{i}$ as query image; and $R_{j}=\left\{i m g_{j_{1}}, i m g_{j_{2}}\right.$, $\left.\ldots, i m g_{j_{N}}\right\}$ the ranked list of reference image $i m g_{j}$. On this way, the axis of context image $\hat{I}$ are ordered according to the order defined by ranked lists $R_{i}$ and $R_{j}$. Let $i m g_{i_{x}} \in R_{i}$ be an image at $x$ postion of ranked list $R_{i}$ and $i m g_{j_{y}} \in R_{j}$ an image at $y$ position of the ranked list $R_{j}$, the value of $f(x, y)$ (function that defines the gray scale of pixel $p(x, y))$ is defined as follows: $f(x, y)=\bar{\rho}\left(i m g_{i_{x}}, i m g_{j_{y}}\right)$, where $\bar{\rho}$ is defined by the distance function $\rho$ normalized in the interval $[0,255]$. 


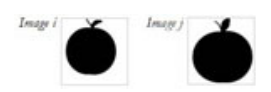

Fig. 1. Similar reference images

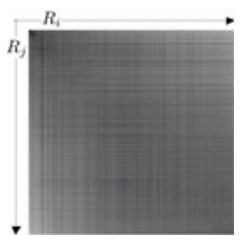

Fig. 3. Representation for similar images

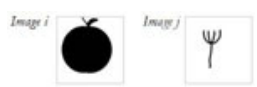

Fig. 2. Dissimilar reference images

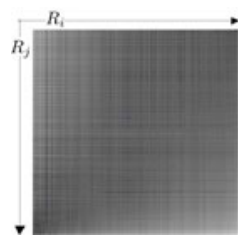

Fig. 4. Representation for dissimilar images

An example considering similar reference images (from MPEG-7 database) is illustrated in Figure 1. The respective gray scale image representing matrix $A$ is illustrated in Figure 3. An analogous example for dissimilar images is illustrated in figures 2 and 4 .

Our goal is to exploit useful context information provided by these images. Low distance values (similar images) are associated with dark pixels in the image, while high values (non-similar images) refers to non-black pixels. Considering two similar images as reference images, the begining of two ranked lists should have similar images as well. This behavior creates a dark region in top left corner of context image (as we can observe in Figure 3). The region represents a neighborhood of similar images with low distances.

We aim to characterize contextual information by analyzing this region using image processing techniques. These information will be used by the re-ranking method presented in next section.

\subsection{Exploiting Contextual Information for Image Re-ranking}

Given an image $i m g_{i} \in \mathcal{C}$, we aim to process contextual information of $\mathrm{img}_{i}$ by constructing context images for the $k$-nearest neighbors of img $_{i}$ (based on distance matrix $A$ ). We use an affinity matrix $W$ to store the results of contextual information. Let $N$ be the size of collection $\mathcal{C}$, the affinity matrix $W$ is a $N \times N$ matrix where $W[k, l]$ represents the similarity between $k$ and $l$.

We apply image processing techniques to process the context image of each $k$-nearest neighbor of $\mathrm{img}_{i}$ and then update the affinity matrix $W$. The same process is performed for all $i m g_{i} \in \mathcal{C}$. Since all images of $\mathcal{C}$ are processed, the affinity matrix $W$ is used as input for computing a new distance martix $A_{t+1}$ (where $t$ indicates the current iteration). These steps are repeated along several iterations. Finally, after a number $T$ of iterations a re-ranking is performed based on final distance matrix $A_{T}$. Algorithm 1 outlines the re-ranking method.

The affinity matrix $W$ is initilized with value 1 for all positions in step 4 . Context images are created in step 7, as explained in Section 2.1, considering 


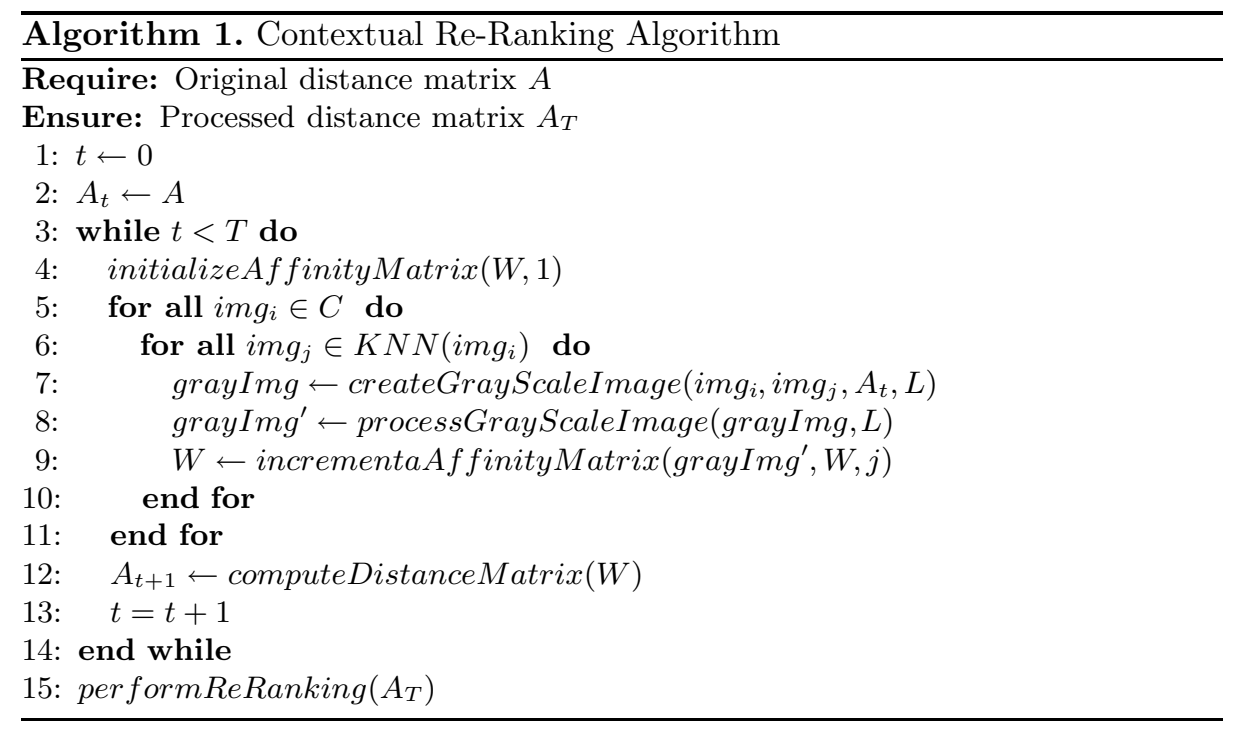

$i m g_{i}$ (image being processed) and $i m g_{j}$ (current neighbor of $i m g_{i}$ ) as reference images. Parameter $L$ refers to the size of the square in top left corner of context image that will be analyzed.

Image processing techniques are applied to context images in step 8. Our goal is to identify dense regions of dark pixels (low distance values). We use a limiarization for obtaining a binary image and discriminating dark pixels. In the following, we apply a median filter for determining regions of dense black pixels. The threshold $l$ used for limiarization is computed based on average and maximum distance values contained in $L \times L$ square in top left corner of context image: $l=\frac{\operatorname{avg}\left(\rho\left(i m g_{i}, i m g_{j}\right)\right)}{\max \left(\rho\left(i m g_{i}, i m g_{j}\right)\right)}$ with $i, j<L$. Figure 5 illustrates an example of limiarized image and Figure 6] shows the limiarized image after applying the median filter.

Step 9 updates the affinity matrix $W$ based on context images. For updating, only black pixels (and their positions) are considered. The idea consists of giving more relevance to pixels next to the origin $(0,0)$, e.g., pixels that represent the begining of ranked lists. The importance of neighbors should also be considered: neighbors at first $k$ positions should be considered more relevant when updating $W$.

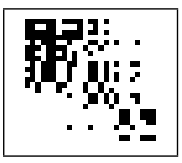

Fig. 5. Example of limiarized image

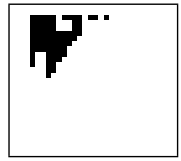

Fig. 6. Example of filtered image

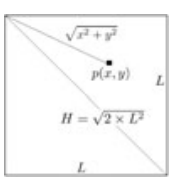

Fig. 7. Computing updates of matrix $W$ 
Let $i m g_{i} \in \mathcal{C}$ be the current image being processed. Let $i m g_{j}$ be the $k$ (such that $k<K$ ) neighbor of $i m g_{i}$. Let $i m g_{i}$ and $i m g_{j}$ be reference images and let $\hat{I}\left(D_{I}, f\right)$ be the context image after limiarization and applying median filter. Let $L$ be the size of top left corner square that should be processed and let $p(x, y) \in D_{I}$ be a black pixel $(f(x, y)=0)$, such that $x, y<L$. Let $H=\sqrt{2 \times L^{2}}$ be the maximum distance of a pixel $p(x, y)$ to origin $(0,0)$, as ilustrated in Figure 7] Let $W\left[i m g_{i_{x}}, i m g_{i_{y}}\right]$ represent the similarity between images $i m g_{i_{x}}$ and $i m g_{i_{y}}$. Then, for each black pixel $p(x, y)$ the matrix $W$ is updated as follows:

$$
W\left[i m g_{i_{x}}, i m g_{i_{y}}\right]=W\left[i m g_{i_{x}}, i m g_{i_{y}}\right]+\left[(K-k) \times\left(H / \sqrt{x^{2}+y^{2}}\right)\right]
$$

Note that low values of $k, x, y$ (the begining of ranked lists) leads to high increments of $W$. Smaller increments occur when $k$ has high values and $x, y=L$. In this case, the term $H / \sqrt{x^{2}+y^{2}}$ is equal to 1 .

At the end of an iteration (when all images have been processed), $W$ presents high values for similar images. But there may be positions of $W$ that did not receive any increments (e.g., dissimilar images), and have the initial value 1 . The new distance matrix $A_{t+1}$ (step 12 of Algorithm 11) is computed as follows:

$$
A_{t+1}[x, y]=\left\{\begin{array}{cc}
1+\bar{A}_{t}[x, y] & \text { if } \mathrm{W}[\mathrm{x}, \mathrm{y}]=1 \\
2 \times(1 / W[x, y]) & \text { if } \mathrm{W}[\mathrm{x}, \mathrm{y}]>1
\end{array}\right.
$$

where $\bar{A}_{t}$ is the distance matrix $A_{t}$ normalized in the interval $[0,1]$. When $W[x, y]=1$, e.g., $W[x, y]$ was not updated by equation 1, we use the old distance matrix $A_{t}$ for determining values of $A_{t+1}$. Otherwise (when $W[x, y]>1$ ), values of new distance matrix $A_{t+1}$ is equal to inverse of affinity matrix $W$. Since the smaller increment for $W$ is 1 (and therefore $W[x, y]=2$ ), the largest value of a new distance in $A_{t+1}$ is 0.5 . Therefore we normalize distance values in the interval $[0,1]$ by multiplying by $2 . A_{t+1}$ will have values in the interval $[0,2]:(i)$ in the interval $[0,1]$ when $W[x, y]>1$, and $(i i)$ in the interval $[1,2]$ when $W[x, y]=1$. A last operation is performed on new distance matrix $A_{t+1}$ for ensure the simetry in terms of distances between images $(\rho(x, y)=\rho(y, x))$ : $A_{t+1}[x, y]=A_{t+1}[y, x]=\min \left(A_{t+1}[x, y], A_{t+1}[y, x]\right)$.

At the end of $T$ iterations, a new computed distance matrix $A_{T}$ is obtained. Finally, a re-ranking is performed based on values of $A_{T}$ (step 15 of Algorithm 1).

\section{Experimental Evaluation}

\subsection{Impact of Parameters}

The execution of Algorithm 1 considers three parameters: (i) $K$ - number of neighbors used as reference images; (ii) $L$ - size of top left square of context image to be analyzed; and (ii) $T$ - number of iterations that the algorithm is executed.

To evaluate the influence of different parameter settings on the retrieval scores and for determining the best parameters values we conducted a set of experiments. We use MPEG-7 database with the so-called bullseye score, which counts 
all matching objects within the 40 most similar candidates. Since each class consists of 20 objects, the retrieved score is normalized with the highest possible number of hits. For distance computation, we used the CFD 9 shape descriptor.

Retrieval scores are computed ranging parameters $K$ in the interval $[1,10]$ and $L$ in the interval $[1,60]$ (with increments of 5 ) for each iteration. For each iteration, the best retrieval score was determined.

We observed that best retrieval scores increased along iterations and parameters converged for values $K=4$ and $L=25$. The best retrieval score was reached in iteration $4: 94.55 \%$. Note that these parameters may change for database with different sizes.

\subsection{Experimental Results}

In this section, we present a set of conducted experiments for demonstrating the applicability and effectiveness of our method. We analyzed our method with respect its use fo re-ranking images considering shape, color, and texture descriptors. We also compared our method to state-of-the-art post-processing methods.

Table 1 presents results (bullseye score - Recall@40) for shape descriptors on MPEG-7 database. We can observe a significative gains from $+5.09 \%$ to $+11.99 \%$.

Table 1. Contextual Re-Ranking for Shape Descriptors on MPEG-7 (Recall@40)

\begin{tabular}{|l|l|l|l|}
\hline Shape Descriptor & Score [\%] & Contextual Re-Ranking & Gain \\
\hline \hline SS [1] & $43.99 \%$ & $49.08 \%$ & $+11.57 \%$ \\
\hline BAS [1] & $75.20 \%$ & $80.35 \%$ & $+6.85 \%$ \\
\hline IDSC+DP [7] & $85.40 \%$ & $89.75 \%$ & $+5.09 \%$ \\
\hline CFD [9] & $84.43 \%$ & $94.55 \%$ & $+11.99 \%$ \\
\hline
\end{tabular}

In addition to shape descriptors, we conducted experiments with color and texture descriptors. For texture descriptor, we used the Brodatz 2 dataset, a popular dataset for texture descriptors evaluation. For color descriptor, we used a soccer data set proposed in [16] and composed by images from 7 soccer teams, containing 40 images per class. For Brodatz dataset, we used the same parameter of MPEG-7 (determined in previous section). Since the soccer dataset have a very different size, we used $K=1, L=50$, and $T=2$. Table 2 presents results for 10 image descriptors in 3 different datasets. The measure adopted is Mean Average Precision (MAP). We can observe that the proposed re-ranking method presented positive precision gains for all descriptors, ranging from $+0.87 \%$ to $+14.75 \%$.

Finally, we also evaluated our method in comparison to other state-of-theart post-processing methods. We use MPEG-7 database with the called bullseye score. Table 3 presents results of our contextual re-ranking method and four postprocessing methods. We also present the retrieval scores for IDSC+DP [7] and CFD 9] shape descriptors, that has been used as input for these methods. Note 
Table 2. Contextual Re-Ranking Evaluation on Content-Based Image Retrieval Tasks

\begin{tabular}{|c|c|c|c|c|c|}
\hline Descriptor & Type & Dataset & $\begin{array}{l}\text { Score [\%] } \\
(M A P)\end{array}$ & $\begin{array}{l}\text { Contextual } \\
\text { Re-Ranking } \\
\end{array}$ & Gain \\
\hline \begin{tabular}{l|l} 
SS [1] \\
\end{tabular} & Shape & MPEG-7 & $37.67 \%$ & $43.23 \%$ & $+14.75 \%$ \\
\hline \begin{tabular}{|l|} 
BAS [1] \\
\end{tabular} & Shape & MPEG-7 & $71.52 \%$ & $75.88 \%$ & $+6.09 \%$ \\
\hline IDSC+DP [7] & Shape & MPEG-7 & $81.70 \%$ & $85.65 \%$ & $+4.83 \%$ \\
\hline CFD [9] & Shape & MPEG-7 & $80.71 \%$ & $91.28 \%$ & $+13.09 \%$ \\
\hline GCH [14] & Color & Soccer Dataset & $32.24 \%$ & $32.52 \%$ & $+0.87 \%$ \\
\hline $\begin{array}{ll}\mathrm{ACC}[3] \\
\end{array}$ & Color & Soccer Dataset & $37.23 \%$ & $39.05 \%$ & $+4.89 \%$ \\
\hline BIC [13] & Color & Soccer Dataset & $39.26 \%$ & $41.81 \%$ & $+6.50 \%$ \\
\hline LBP [8] & Texture & Brodatz & $48.40 \%$ & $49.59 \%$ & $+2.46 \%$ \\
\hline CCOM [6] & Texture & Brodatz & $57.57 \%$ & $63.48 \%$ & $+10.27 \%$ \\
\hline LAS [15] & Texture & Brodatz & $75.15 \%$ & $78.39 \%$ & $+4.31 \%$ \\
\hline
\end{tabular}

Table 3. Post-processing methods comparison on MPEG-7 database (Recall@40)

\begin{tabular}{|l|l|l|l|}
\hline Algorithm & Descriptor & Score [\%] & Gain \\
\hline \hline CFD [9] & - & $84.43 \%$ & - \\
\hline IDSC+DP [7] & - & $85.40 \%$ & - \\
\hline \hline Graph Transduction [17] & IDSC+DP & $91.00 \%$ & $+6.56 \%$ \\
\hline Distance Optmization [9] & CFD & $92.56 \%$ & $+9.63 \%$ \\
\hline Constrained Diffusion Process [18] & IDSC+DP & $93.32 \%$ & $+9.27 \%$ \\
\hline Mutual kNN Graph [5] & IDSC+DP & $93.40 \%$ & $+9.37 \%$ \\
\hline Contextual Re-Ranking & CFD & $\mathbf{9 4 . 5 5 \%}$ & $+\mathbf{1 1 . 9 9 \%}$ \\
\hline
\end{tabular}

that our contextual re-ranking method (last line) has the best effectiveness performace when compared to all other post-processing methods.

\section{Conclusions}

In this work, we presented a new re-ranking method based on contextual information. The main idea consists in creating gray scale image representations of distance matrix and performs a re-ranking based on information extracted from these images. We conducted a large set of experiments and experimental results demonstrated the applicability of our method to several image retrieval tasks based on shape, color and texture descriptors. The proposed method achieves very high effectiveness performance when compared with state-of-the-art postprocessing methods on the well-known MPEG-7 dataset. Future work focuses on using other image processing techniques, as dynamic limiarization and filtering.

\section{Acknowledgment}

Authors thank CAPES, FAPESP and CNPq for financial support. Authors also thanks DGA/UNICAMP for its support in this work. 


\section{References}

1. Arica, N., Vural, F.T.Y.: Bas: a perceptual shape descriptor based on the beam angle statistics. Pattern Recogn. Lett. 24(9-10), 1627-1639 (2003)

2. Brodatz, P.: Textures: A Photographic Album for Artists and Designers. Dover, New York (1966)

3. Huang, J., Kumar, S.R., Mitra, M., Zhu, W.J., Zabih, R.: Image indexing using color correlograms. In: CVPR 1997, p. 762 (1997)

4. Jégou, H., Harzallah, H., Schmid, C.: A contextual dissimilarity measure for accurate and efficient image search. In: CVPR, pp. 1-8 (2007)

5. Peter, K.: Donoser Michael, B.H.: Beyond pairwise shape similarity analysis. In: Asian Conference on Computer Vision (ACCV), pp. 655-666 (2009)

6. Kovalev, V., Volmer, S.: Color co-occurence descriptors for querying-by-example. In: MMM 1998, p. 32 (1998)

7. Ling, H., Jacobs, D.W.: Shape classification using the inner-distance. PAMI 29(2), 286-299 (2007)

8. Ojala, T., Pietikäinen, M., Mäenpää, T.: Multiresolution gray-scale and rotation invariant texture classification with local binary patterns. PAMI 24(7), 971-987 (2002)

9. Pedronette, D.C.G., da Torres, R.S.: Shape retrieval using contour features and distance optmization. In: VISAPP. vol. 1, pp. 197 - 202 (2010)

10. Perronnin, F., Liu, Y., Renders, J.M.: A family of contextual measures of similarity between distributions with application to image retrieval. In: CVPR, pp. 2358-2365 (2009)

11. da, S., Torres, R., Falcao, A.X.: Contour Salience Descriptors for Effective Image Retrieval and Analysis. Image and Vision Computing 25(1), 3-13 (2007)

12. Schwander, O., Nielsen, F.: Reranking with contextual dissimilarity measures from representational bregmanl k-means. In: VISAPP, vol. 1, pp. 118-122 (2010)

13. Stehling, R.O., Nascimento, M.A., Falcao, A.X.: A compact and efficient image retrieval approach based on border/interior pixel classification. In: CIKM 2002, pp. 102-109 (2002)

14. Swain, M.J., Ballard, D.H.: Color indexing. IJCV 7(1), 11-32 (1991)

15. Tao, B., Dickinson, B.W.: Texture recognition and image retrieval using gradient indexing. JVCIR 11(3), 327-342 (2000)

16. van de Weijer, J., Schmid, C.: Coloring local feature extraction. In: Leonardis, A., Bischof, H., Pinz, A. (eds.) ECCV 2006, Part II. LNCS, vol. 3952, pp. 334-348. Springer, Heidelberg (2006)

17. Yang, X., Bai, X., Latecki, L.J., Tu, Z.: Improving shape retrieval by learning graph transduction. In: Forsyth, D., Torr, P., Zisserman, A. (eds.) ECCV 2008, Part IV. LNCS, vol. 5305, pp. 788-801. Springer, Heidelberg (2008)

18. Yang, X., Koknar-Tezel, S., Latecki, L.J.: Locally constrained diffusion process on locally densified distance spaces with applications to shape retrieval. In: CVPR, pp. 357-364 (2009) 